\title{
Morphology, microstructure and magnetic properties of spark-eroded ferromagnetic Ni-Mn-Ga fine particles
}

\author{
Virgil C. Solomon,* Martha R. McCartney,** YunJun Tang, ${ }^{* *}$ Ami E. Berkowitz, ${ }^{* * *}$ and \\ David J. Smith *,** \\ * Center for Solid State Science, Arizona State University, Tempe, AZ 85287-1704 \\ ** Department of Physics and Astronomy, Arizona State University, Tempe, AZ 85287-1504 \\ *** Center for Magnetic Recording Research, University of California at San Diego, La Jolla, CA \\ 92093-0401
}

Ni-Mn-Ga alloys show large reversible linear strain of up to $9.5 \%$ under an applied magnetic field. This behavior is attributed to the rearrangement of twin-related martensite variants, which are crystallographically equivalent but with different orientation [1]. Practical applications have some drawbacks, due to poor mechanical characteristics of single crystals, but these can be overcome by designing particle-polymer composites. Fine Ni-Mn-Ga particles suitable for use in preparing composite materials were produced by spark-erosion in liquid nitrogen and liquid argon [2]. The objective of the present investigation is to clarify the morphology, microstructure and magnetic properties of ferromagnetic $\mathrm{Ni}_{50.7} \mathrm{Mn}_{29.5} \mathrm{Ga}_{19.8}$ (at.\%) spark-eroded particles.

The particles are nearly spherical in shape, irrespective of the dielectric used in the spark-erosion process (Fig.1). Investigation of cross-sectioned samples, revealed the main morphological differences between spark-eroded particles prepared in liquid argon and liquid nitrogen, see Figs. 1 (c) and (d), respectively. At room temperature, the microstructure of ferromagnetic particles consisted of twinned martensite, as shown in Fig. 2(a). The martensite has an approximately tetragonal lattice with $\mathrm{c} / \mathrm{a}=0.94$ as confirmed by electron diffraction. Selected-area electron diffraction pattern in Fig. 2(b) shows superlattice spots at 1/5 positions along the [110]* direction of the reciprocal lattice (five-layered martensite). The projection of c-axis, which is the easy axis of magnetization for this material, on the image plane is indicated in Fig. 2(c).

Investigations of magnetic properties of Ni-Mn-Ga spark-eroded particles have been carried out by using Lorentz microscopy and electron holography. The Lorentz image in Fig. 3(a) shows the coincidence of twin boundaries with magnetic domain walls. Figure 3(b) shows the reconstructed phase image obtained from the electron hologram of a twinned area. The in-plane projection of the magnetization vectors are orientated at roughly $60^{\circ}$ to each other, as indicated in Fig. 3(c) by the overlapping color-scale phase and induction maps. This orientation corresponds to a calculated angle of $86.5^{\circ}$ between c-axes of the twin-related variants when projected onto the image plane. The calculated values of magnetization (along the easy c-axis), and magnetic domain wall thickness, are about $0.57 \mathrm{~T}$ and $17 \mathrm{~nm}$, respectively [3].

\section{References}

[1] R. C. O'Handley, J. Appl. Phys. 83 (1998) 3263.

[2] A. E. Berkowitz et al., Appl. Phys. Lett. 85 (2004) 940.

[3] This work was supported by ONR Grant No. N00014-1-0758. We acknowledge use of the facilities in the John M. Cowley Center for High Resolution Electron Microscopy at ASU. 

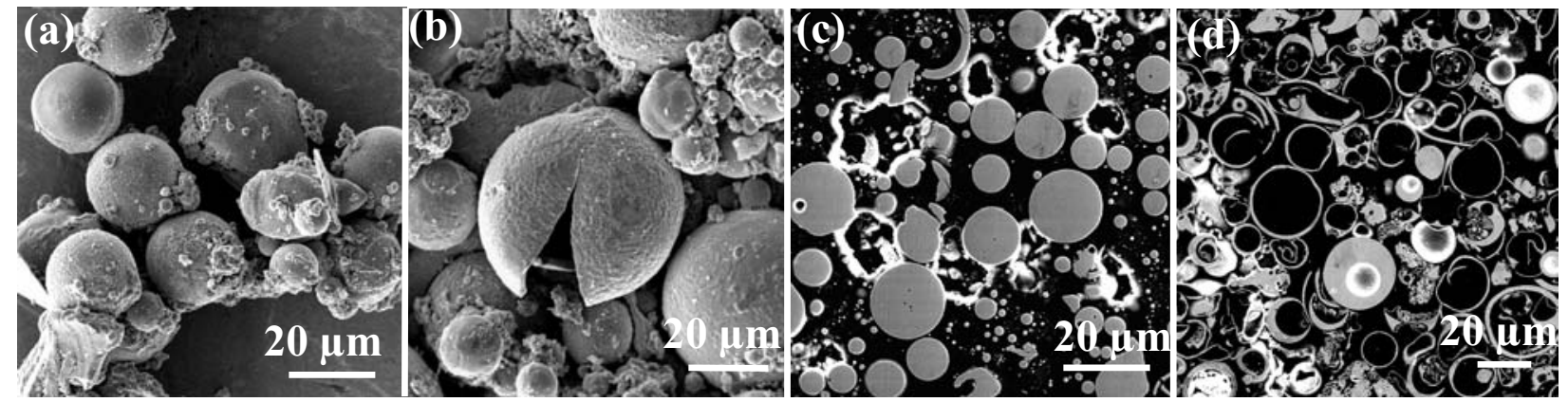

Fig. 1. SEM micrographs of $\mathrm{Ni}_{50.7} \mathrm{Mn}_{29.5} \mathrm{Ga}_{19.8}$ spark-eroded particles in (a) liquid argon and (b) liquid nitrogen. (c) and (d) Images of cross-sectioned samples showing solid spheres and thin-walled particles obtained by spark-erosion in liquid argon and liquid nitrogen, respectively.
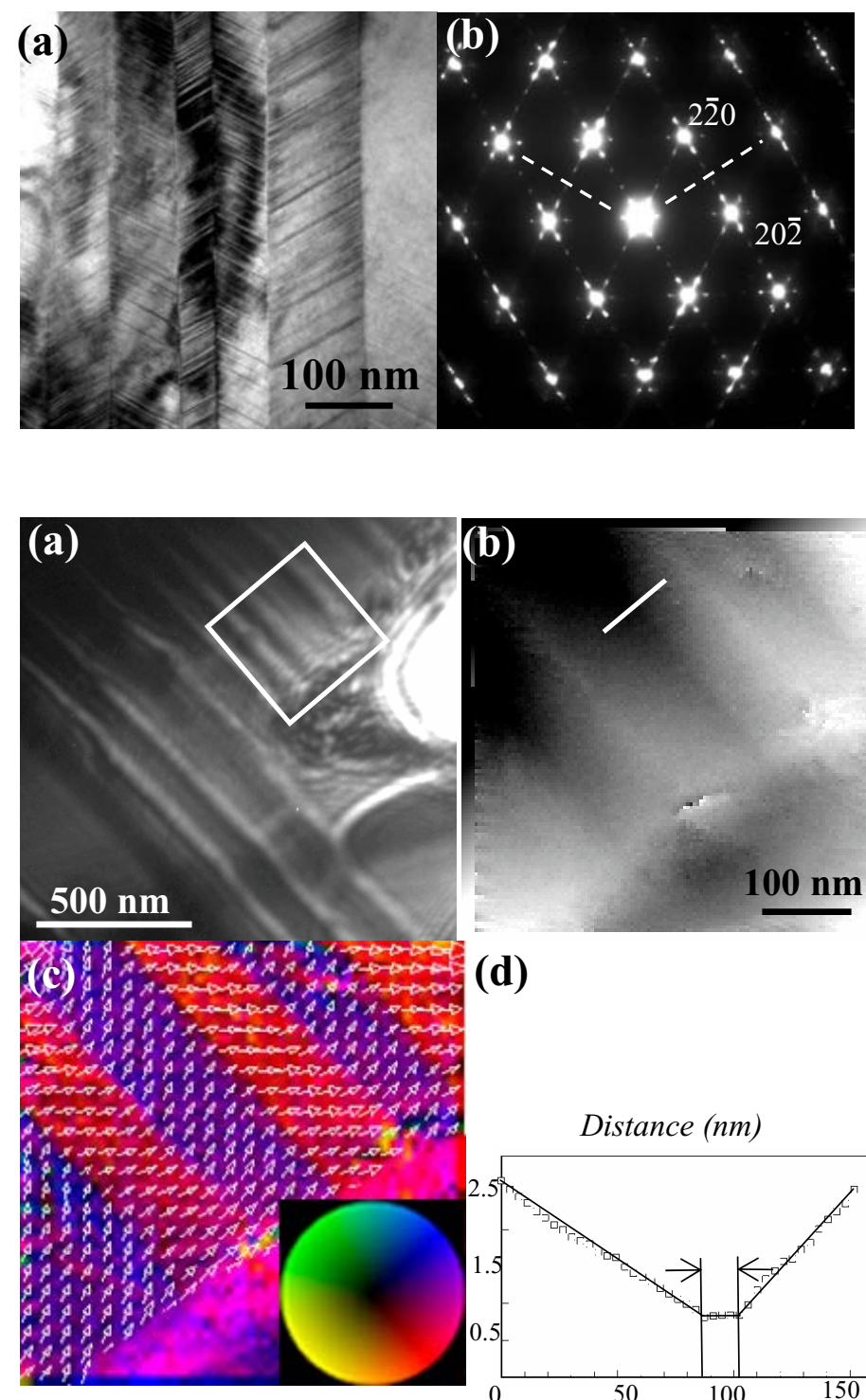

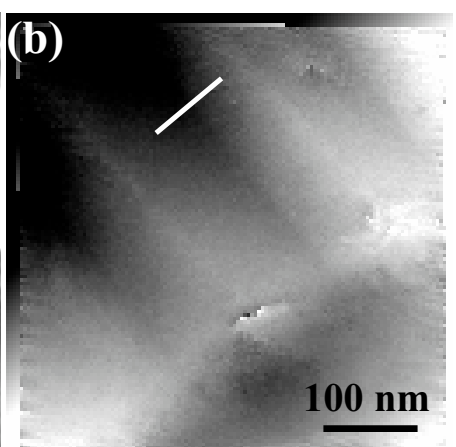

(d)

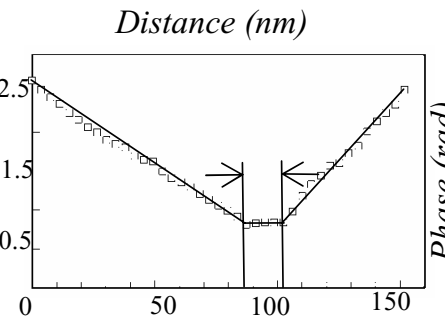

Fig. 2. (a) Bright field image showing typical twinned marteniste microstructure of ferromagnetic $\mathrm{Ni}_{50.7} \mathrm{Mn}_{29.5} \mathrm{Ga}_{19.8}$ sparkeroded particles at room temperature. (b) Selected-area electron diffraction pattern of [111] zone taken from the area shown in (a). The dashed lines indicate the easy axis projection onto specimen plane.

Fig.3. (a) Under-focus Lorentz image of a twinned microstructure of Ni-Mn-Ga spark-eroded particles. (b) Phase image reconstructed from the electron hologram of the area indicated in (a). (c) Color map with superimposed vector map obtained from phase gradient image showing change of magnetization direction across domain walls. (d) Domain wall width can be determined using phase image by measuring the width of the region over which the phase slope changes abruptly. From the line profile, corresponding to the white segment in (b), the domain wall width was estimated to be about $17 \mathrm{~nm}$. 